\title{
IN SILICO ANALYSIS OF ACTIVE CONSTITUENTS OF SILYMARIN AS ALPHA-GLUCOSIDASE ENZYME INHIBITORS IN TYPE 2 DIABETES MELLITUS
}

\author{
AHMAD ZONOUBI ${ }^{1 *}$, PRASHANTHA CN ${ }^{2}$, D VISAGA PERUMAL ${ }^{1}$, ZAHRA MAFIBANIASADI ${ }^{3}$ \\ ${ }^{1}$ Department of Pharmaceutical Chemistry, T. John College of Pharmacy, Bengaluru, Karnataka, India. ${ }^{2}$ Department of Biotechnology, \\ School of Applied Sciences, REVA University, Bengaluru, Karnataka, India. ${ }^{3}$ Department of Pharmaceutics, T. John College of Pharmacy, \\ Bengaluru, Karnataka, India. Email: ahmad.zonoubi84@gmail.com
}

Received: 05 June 2019, Revised and Accepted: 25 July 2019

ABSTRACT

Objective: Type 2 diabetes mellitus (T2DM) is an acute metabolic disorder, in which the vogue is increasing persistently globally. The maltase-glucoamylase/alpha-glucosidase inhibitor is an oral antidiabetic drug collectively, which is utilizing for regulating carbohydrates that ordinarily transformed into simple sugars and absorbed by the intestine. Researchers need to constantly explore alternative therapeutic strategies for the clinical management of DM due to the increased adverse event caused by conservative antidiabetic agents. The present study proposes a substitute drug to examine the seven bioactive phytocomponents of Silybum marianum (milk thistle) that can regulate the hyperglycemia by downregulating alpha-glucosidase and its activity.

Methods: Different integrated web-based in silico tools and techniques were used to model the enzyme (receptor) as well as to determine the druggability of different active constituents of silymarin and their pharmacokinetics were predicted. Further, the active site of the enzyme was predicted followed by molecular docking method.

Results: The results show silychristin A and silydianin having less carcinogenicity and strong interaction to the target protein (alpha-glucosidase) compare to the reference drugs (acarbose and miglitol) and these two molecules can be used for the best drug molecules in T2DM.

Conclusion: In the proposed study, the in silico analysis helps researchers to utilize these compounds for clinical applications. The conclusion also suggests that synthetically and semi-synthetically, nucleus and peripheral modifications, either in the form of skeletal rearrangements or partial degradations as well as functional group addition and replacement of the active molecules present in silymarin giving access to new structural motifs, which can be used in future as a lead compounds for antagonising the alpha-glucosidase in the treatment of diabetes mellitus.

Keywords: Type 2 diabetes mellitus, Alpha-glucosidase, Silymarin, Silychristin A, Silydianin, Acarbose, Miglitol, Molecular docking, Milk thistle.

(c) 2019 The Authors. Published by Innovare Academic Sciences Pvt Ltd. This is an open access article under the CC BY license (http://creativecommons. org/licenses/by/4. 0/) DOI: http://dx.doi.org/10.22159/ajpcr.2019.v12i9.34460

\section{INTRODUCTION}

Diabetes mellitus (DM) is undoubtedly one of the human's oldest known diseases. It was first reported about 3000 years ago in the Egyptian manuscript [1]. A perfect variance among T1 and Type 2 DM (T2DM) was undoubtedly defined [2,3]. T2DM was previously illustrated in 1988 as a component of metabolic syndrome [4]. The prompts of T2DM are complex, multifaceted and comprise both genetic and environmental features as well as behavioral risk factors that affect beta-cell obligation and tissue insulin sensitivity [5-7]. Persons with T2DM are more susceptible to various forms of short-term and long-term medical difficulties, which occasionally lead to premature death. This has been seen in patients with T2DM mostly due to the pervasiveness of this type of DM. As the prevalence of this metabolic disorder, it is promptly rising and standard treatment refuses to steady the disease in most patients, prevention would be perceived as a key objective in the relatively proximate future. Persons who experience T2DM go through a phase of impaired glucose tolerance (IGT). Defects in the action or even secretion of insulin seem to be the two main complications ultimately leading to the development of glucose intolerance. Any interference in the phase of IGT that decreases insulin resistance protects beta-cells or both should also prevent or delay progression to diabetes.

Several potential drugs such as sulfonylureas, meglitinides, biguanides, and thiazolidinediones and alpha-glucosidase inhibitors can be used in medical treatments to regulate high blood glucose problems (hyperglycemia). In general, alpha-glucosidase is concomitant through the breakdown of polysaccharide and disaccharides into monosaccharide glucose [8,9]. Alpha-glucosidase inhibitors are oral antidiabetic medications which administered to alter the breakdown of carbohydrates that are transformed into simple sugars and absorbed by the intestines [10]. Alpha-glucosidase inhibitors appear to have a greater affinity of 10,000-100,000 times with carbohydrate-binding sites than oligosaccharides and polysaccharides. Oligosaccharides and polysaccharides cannot be converted into simple sugars that the body can absorb by inhibition of alpha-glucosidase enzyme [11]. Hereafter, alphaglucosidase inhibitors are crucial medications to prevent the absorption of carbohydrates in the intestine and can be used to treat T2DM and IGT [12]. Thus, these hypoglycemic agents are applied to patients with early diabetes or combined with other drugs [13]. Medicinal plants and herbs are noticeable in traditional medicine and have appealing pharmacological activities [14] and the most significant properties of medicinal plants are, either they show lower side effects or they are without any side effects.. During the past decade, alpha-glucosidase inhibitors from natural resources have been extensively established as highly purposeful foods or lead compounds to modulate diabetes.

Recently, drug designs used to significantly diminish blood glucose levels and try to maintain blood glucose homeostasis with slightly fewer adverse effects. In the structure-based drug design method, structures of considered target proteins are usually utilizing to perceive novel compounds that are therapeutically applicable. Computational or molecular docking is a simulation strategy that predicts molecules that properly bind to targets including enzymes and receptor proteins. Moreover, the rational methodology in virtual screening is known as the hierarchical method, which seems to be a valuable tool for computing 
and screening protein structures to explore new ligands [15]. The ligand must have a high affinity with proteins to assess the binding site of a compound for a given receptor, as well as the property of interactions and leverage based on a scoring entity [16]. For example, the activity of a precise protein in human can be antagonized by the inhibitor discovery and protein-ligand interactions can be chosen mainly as a possible drug development which is called a pharmacophore model that can be utilized to achieve and recognize small molecules in twodimensional or three-dimensional (3D) (in silico) protein structure and function prediction [17]. Therefore, the demand for enhanced pharmacophore modeling is significant to diminish drug costs and new drugs are most likely to bind to the target protein $[18,19]$. The current study conducted a high-performance molecular docking to screen the potential of alpha-glucosidase from natural compounds and evaluated the analysis of alpha-glucosidase activities.

\section{METHODS}

\section{Homology modeling}

The target sequences of alpha-glucosidase enzyme were compiled from the UniProtKB protein knowledgebase (ID: 043451) and compelled using NCBI PSI-BLAST to identify the template sequences. Further, the 3D protein structures were built using Swiss-Model (2QLY.1.A) and the protein structure was modeled using Swisspdbv viewer [20-23]. The consequential 3D structure of the protein was exposed to quality analysis using SAVES to predict the stereochemical activity of amino acids on the bases of geometry through various parameters such as WHATIF, ERRAT, and PROCHECK [24-27].

\section{Active site prediction}

The geometric and topological possessions of modeled protein 2QLY.1.A (Computed Atlas of Surface Protein Topography [CASTp] ID: J_5C744E039BB01) exerted to predict active site amino acid, based on surface pockets, interior cavities, cross-channel, and hydrogen bond interactions using CASTp calculation server. CASTp is a web server which provides online services for retrieving, outlining, and quantifying these geometric and topological attributes of protein structures [28]. The basic components of computational geometry exert in CASTp are Delaunay triangulation, alpha shape, and discrete flow [29-33]. The modeled 2QLY.1.A (CASTp ID: J_5C744E039BB01) active sites were revealed using CASTp web server.

\section{Ligand preparation and pharmacophore analysis}

Silybin A, silybin B, isosilybin A, isosilybin B, silychristin A, silychristin B, and silydianin are active components (phytoconstituents) present in milk thistle that was used as an assessment compounds, and acarbose and miglitol as a standard compound were retrieved from ChEMBL Database using SDF file format (https://www.ebi.ac.uk/chembl/). Moreover, the structures of the ligands (active molecules) were converted to Mol. format using Chemsketch software to avoid any unsolicited mistake in the structures. The pharmacophore analysis was performed to determine chemical properties and predict drug-like molecule, by means of molinspiration online server. The rule of 5 shows the considerations which are a set of basic molecular descriptors to predict drug-like molecule which have Log $p \leq 5$, molecular weight (MW) $\leq 500$, hydrogen bond acceptor numbers $\leq 10$, and hydrogen bond donor numbers $\leq 5$. Those molecules which violate rather than one of these rules might have bioavailability problems [34]. These parameters help to screen the compounds to understand drug-like properties.

\section{Pharmacokinetic properties}

Pharmacokinetic properties of different components of the silymarin were calculated using an admetSAR online server. Calculation of physicochemical parameters of small molecules is valuable to screen computationally of the chemical compounds for their druglikeness and even toxicity potential. The overall of Log P, topological polar surface area (TPSA), H-bond acceptor, H-bond donors, and MW were worked out separately for an individual constituent by means of OpenBabel v2.3.1 [35] that can be utilized for absorption, distribution, metabolism, excretion, and toxicity (ADMET) prediction. The incipience of initial screening for ADMET has augmented for drug nominees incept in the drug discovery phase and decreased the percentage of compounds that failed in clinical trials due to ADMET reasons. The TPSA calculation has relied on the summation of the fragment contributions, which regards the polar fragments centered on $\mathrm{O}$ and N. Polar surface area is revealed to be an admirable descriptor for drug absorption such as intestinal absorption, bioavailability, Caco-2 permeability, and blood-brain barrier penetration [36]. The measurement of molecular flexibility is the number of rotatable bonds (Nrotb), which is the ordinary topological parameter. The Nrotb is a good descriptor to measure oral drug bioavailability [37]. Single non-ring bond, bound to the nonterminal heavy atom (i.e., non-hydrogen), is considered to be a rotatable bond. Amide C-N bonds are not considered due to their high rotational energy barrier.

\section{Molecular docking}

Based on the pharmacophore and pharmacokinetic analysis, the designated compounds were docked with the target protein (alphaglucosidase) using MGL Tools 1.5.6 and AutoDock 4.2; (Autodock, Autogrid, Copyright-1991e2000) from the Scripps Research Institute, http:/www.scripps.edu/mb/olson/doc/autodock so that to start and run AutoDock, polar hydrogens were added to the protein alongside with the Gasteiger type and the non-polar hydrogen was merged with the carbons. After that, the internal degrees of freedom and torsion were determined to prepare flexible and rigid molecule and to prepare grid maps of different grid points that keep ligands covering binding pockets fully based on active site amino acids within the selected proteins. The size of the grid box was adjusted based on $\mathrm{X}, \mathrm{Y}$, and Z axis and $70 \times 70 \times 70$ further require to calculate grid parameters using Autogrid. Molecular simulation parameters further to calculate Autodock properties to understand protein-ligand interactions by adding the Lamarckian genetic algorithm and molecular simulation methods to evaluate molecular docking. Though all parameters were considered necessary for molecular docking and therefore, pharmacophore mapping were fixed and used regularly for different compounds [38-40]. Consequently, the output of the docking process was analyzed utilizing EduPymol version 1.7.4.4.

\section{RESULTS AND DISCUSSION}

The selected alpha-glucosidase protein sequences were aligned using PSI-BLAST and modeled using modeler. The resultant protein structure is built using Swisspdb viewer. The results showed alpha-glucosidase protein has $99.77 \%$ of similarity to $5 \mathrm{KZW} \_$A protein template with the e-value of 0.0 and total score of 1798 . The quality analysis of the modeled protein was predicted using SAVES. The modeled alpha-glucosidase protein has a similar template of $99.77 \%$ similar to alpha-glucosidase with e-value of 0.0 . We built the modeled structure and observed the Ramachandran plot with amino acids in the allowed region that shows $85.6 \%$ of amino acids are in complex prediction (Fig. 1) that it shows the

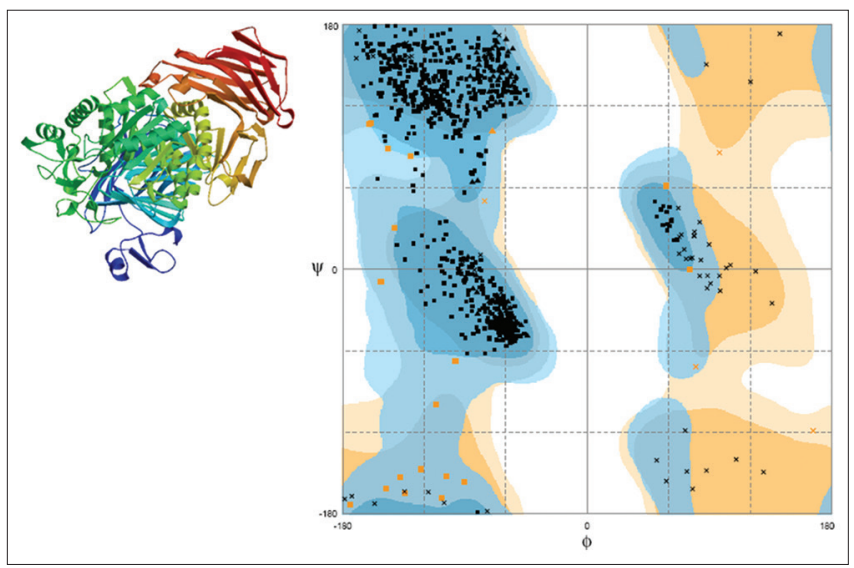

Fig. 1: $\alpha$-Glucosidase protein structure is modeled by Swiss-Model and Ramachandran plot was predicted using RAMAPAGE 
rigidity of the protein structure. The ligand-binding site was observed using CASTp based on the pocket region of geometrically distributed amino acids and based on interaction energy. Some of the active site amino acids such as TYR385, ASP413, ILE414, MET417, ARG420, ASP422, ILE450, ASP452, PR0453, ALA454, GLU490, VAL491, TRP492,

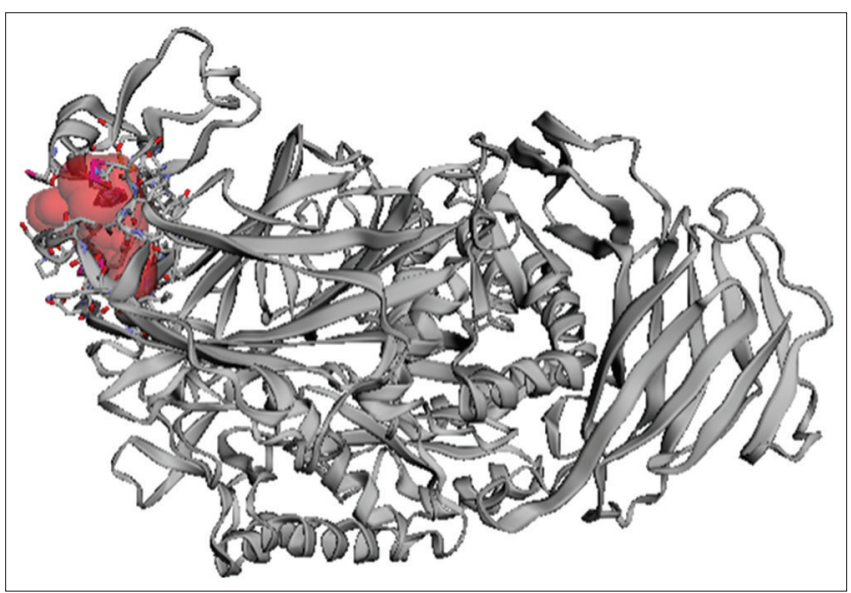

Fig. 2: $\alpha$-Glucosidase protein structures are used to predict ligand-binding sites and pockets using CASTp calculation server
PR0493, PHE498, TRP527, ASP529, MET530, VAL533, SER534, ASN535, PHE536, ARG612, TRP625, ASP628, ASP657, PHE661, and HIS686 amino acid residues were found to be the best binding sites (Fig. 2). These active sites were considered for molecular docking.

\begin{abstract}
Ligand structure preparation and pharmacophore analysis
Chemical library database such as chEMBL was used to retrieve the bioactive compounds along with reference molecules. The ligand structures were screened to understand drug-like properties (Tables 1-3). ADMET and toxicity were performed employing Molinspiration and admetSAR online tools. The result obtained shows that the standard drugs having the higher predicted value of carcinogenicity, acarbose $=1.00$ and miglitol $=0.9714$ in comparison with silychristin $A=0.9165$ and silydianin $=0.9571$ with the lower value of carcinogenicity. The molecular simulation techniques were utilized to simulate the chemical structure to predict the electrostatic interaction and potential energy prediction. QSAR properties were predicted to the molecular simulated chemical structures utilizing Hyperchem.
\end{abstract}

\section{Molecular docking and virtual screening}

Molecular docking of particular bioactive compounds alongside with reference drug molecules was docked with target protein alphaglucosidase. On the other hand, the docking results were predicted based on the binding energy (kcal/mol), number of the hydrogen bond, inhibitory constant (Ki), ligand efficiency, and interacting amino acids

Table 1: Pharmacophore analysis of silymarin active compounds predicted using Molinspiration

\begin{tabular}{|c|c|c|c|c|c|c|c|c|}
\hline Ligand & miLogP & TPSA & nAtoms & MW & nON & nOHNH & Nrotb & Volume \\
\hline Silybin A & 1.47 & 155.15 & 35 & 482.44 & 10 & 5 & 4 & 400.86 \\
\hline Silybin B & 1.47 & 155.15 & 35 & 482.44 & 10 & 5 & 4 & 400.86 \\
\hline Isosilybin A & 1.47 & 155.15 & 35 & 482.44 & 10 & 5 & 4 & 400.86 \\
\hline Isosilybin B & 1.47 & 155.15 & 35 & 482.44 & 10 & 5 & 4 & 400.86 \\
\hline Silychristin A & 1.26 & 166.14 & 35 & 482.44 & 10 & 6 & 4 & 399.89 \\
\hline Silychristin B & 1.26 & 166.14 & 35 & 482.44 & 10 & 6 & 4 & 399.89 \\
\hline Silydianin & 1.21 & 162.98 & 35 & 482.44 & 10 & 5 & 3 & 395.34 \\
\hline Miglitol & -2.75 & 104.38 & 14 & 207.23 & 6 & 5 & 3 & 189.18 \\
\hline
\end{tabular}

TPSA: Topological polar surface area, nAtoms: Number of atoms, MW: Molecular weight, nON: Number of hydrogen bond acceptor, nOHNH: Number of hydrogen bond donor, Nrotb: Number of rotatable bond

Table 2: Absorption, distribution, metabolism, excretion, and toxicity analysis of silymarin active compounds predicted using admet SAR tool

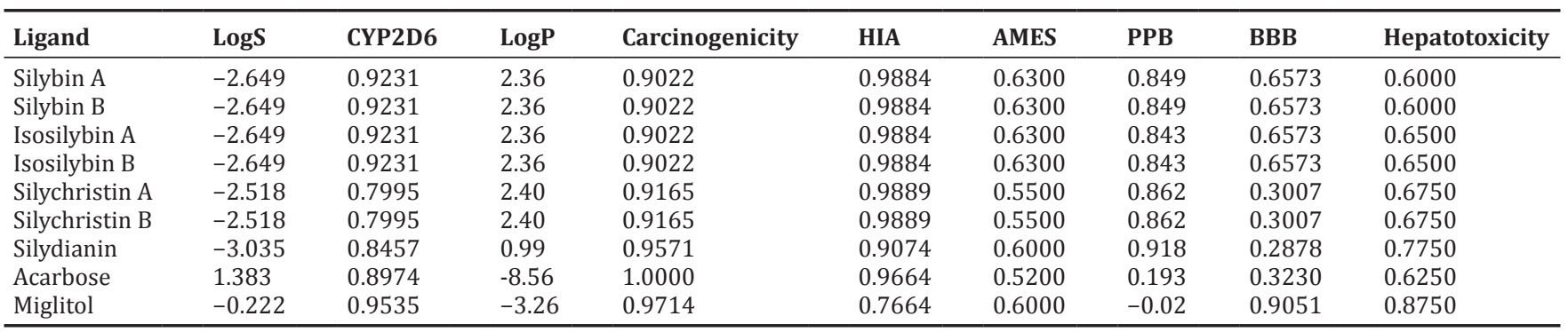

LogS: Solubility measured in mol/liter, HIA: Human intestinal absorption, AMES: Chemical mutagenicity, PPB: Plasma protein binding, BBB: Blood-brain barrier

Table: 3 Molecular docking of silybin active compounds against alpha-glucosidase protein structure using AutoDock

\begin{tabular}{lllll}
\hline Ligand & H-bond & Binding energy (kcal/mol) & IC $_{\text {50 }}$ Ki (uM/nM) & Amino acids \\
\hline Silybin A & 2 & -8.35 & $760.85(\mathrm{nM})$ & ALA454, TYR691 \\
Silybin B & 2 & -7.72 & $2.18(\mathrm{uM})$ & ALA454, GLN689 \\
Isosilybin A & 4 & -8.86 & $320.68(\mathrm{nM})$ & TRP492, GLN689, GLN689, TYR691 \\
Isosilybin B & 3 & -8.06 & $1.24(\mathrm{uM})$ & TYR385, TRP492, TYR691 \\
Silychristin A & 3 & -10.00 & $46.70(\mathrm{nM})$ & TRP492, ARG612, TYR691 \\
Silychristin B & 3 & -9.51 & $106.26(\mathrm{nM})$ & TYR385, ALA454, ASP628 \\
Silydianin & 4 & -9.80 & $65.25(\mathrm{nM})$ & GLU490, SER534, TRP492, ARG612 \\
Acarbose & 3 & -7.19 & $5.41(\mathrm{uM})$ & GLN689, GLN689, GLN689 \\
Miglitol & 5 & -5.60 & $77.96(\mathrm{uM})$ & TRP492, SER534, GLU490, GLU490, SER534 \\
\hline
\end{tabular}




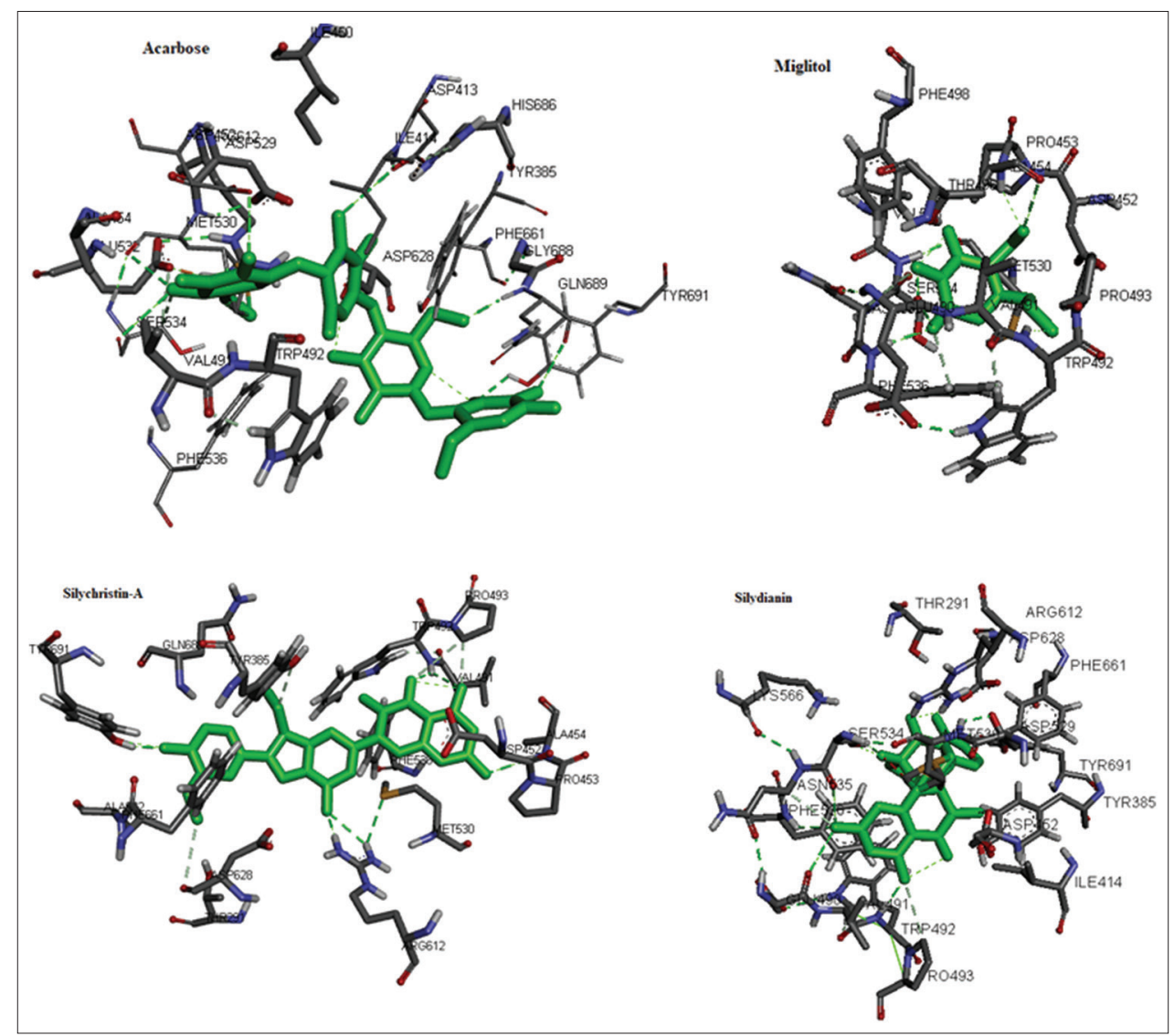

Fig 3: Diagrams of ligand-protein interaction in $\alpha$ - Glucosidase pocket by EduPymol

within the target proteins. Virtual screening of the reference drugs with bioactive compounds was observed based on genetic algorithm cluster histogram table and the overall results are listed in Table 3. The docking results were observed with reference molecules compared with bioactive compounds that show, acarbose has three hydrogen bonds with the binding energy of $-7.19 \mathrm{kcal} / \mathrm{mol}$ and $\mathrm{Ki}=5.41 \mu \mathrm{M}$ and another reference drug miglitol has five hydrogen bonds with the binding energy of $-5.60 \mathrm{kcal} / \mathrm{mol}$ and $\mathrm{Ki}=77.65 \mu \mathrm{M}$. The reference drugs compared with bioactive compounds were shown that silychristin A has three hydrogen bonds with the binding energy of $-10.00 \mathrm{kcal} / \mathrm{mol}$ and $\mathrm{Ki}=46.70 \mathrm{nM}$ and silydianin has four hydrogen bonds with the binding energy of $-9.80 \mathrm{kcal} / \mathrm{mol}$ and $\mathrm{Ki}=65.25 \mathrm{nM}$. Other molecules revealed 2-3 hydrogen bonds with the lowest binding energy with Ki of $\mu \mathrm{M}$ concentrations. The overall result predicts that the silychristin A and silydianin having the best pharmacological characters, drug-like properties, and strong binding interactions based on the concentration of the compound inhibition. The hydrogen-bond interactions and its binding energy were assessed for the finest affinity by means of EduPymol Molecular Viewer software (Fig. 3).

\section{CONCLUSION}

The ADMET profile of the active constitutions of the silymarin has been estimated which showed less carcinogenicity compared with standard drugs. Even more, based on the protein-ligand interaction shows that silychristin A and silydianin having the best interaction compare to the reference drugs (acarbose and miglitol) toward the enzyme (alphaglucosidase). Further investigation has to be done for confirmation and evaluation of these compounds. The conclusion of this study suggested that silychristin A and silydianin having a good biological activity which can consider them as a lead molecule present in milk thistle. In the proposed study, the in silico analysis helps researchers to utilize these compounds for clinical applications. The conclusion also suggests that synthetically and semi-synthetically, nucleus and peripheral modifications, either in the form of skeletal rearrangements or partial degradations as well as functional group addition and replacement of the active molecules present in silymarin giving access to new structural motifs, which can be used in future as a lead compounds for antagonising the alpha-glucosidase in the treatment of diabetes mellitus.

\section{AUTHORS' CONTRIBUTIONS}

Ahmad Zanoubihas designed the protocol, conducting of experiment, data collection, analysis, and preparation of manuscript; Prashantha $\mathrm{C}$ $\mathrm{N}$ and D. Visaga Perumal were guiding for conducting experiment and reviewing of the manuscript; and Zahra Mafibaniasadi collaborating in data collection for pharmacokinetic evaluation in the present study.

\section{CONFLICTS OF INTEREST}

There are no conflicts of interest reported and the work did not involve any financial support from any external agency.

\section{REFERENCES}

1. Lakhtakia R. The history of diabetes mellitus. Sultan Qaboos Univ Med J 2013;13:368-70.

2. López-Cuenca A, Gómez-Molina M, Flores-Blanco PJ, Sánchez-Martínez M, García-Narbon A, De Las Heras-Gómez I, et al. Comparison between Type-2 and type-1 myocardial infarction: Clinical features, treatment strategies and outcomes. J Geriatr Cardiol 2016;13:15-22.

3. Jain R, Jain P, Jain P. A review on treatment and prevention of diabetes mellitus. Int J Curr Pharm Res 2016;8:16-8.

4. Okur ME, Karantas ID, Siafaka PI. Diabetes mellitus: A review on pathophysiology, current status of oral medications and future perspectives. Acta Pharm Sci 2017;55:61-82.

5. Scheen AJ. Pathophysiology of Type 2 diabetes. Acta Clin Belg 2003;58:335-41.

6. Staiger H, Machicao F, Fritsche A, Häring HU. Pathomechanisms of Type 2 diabetes genes. Endocr Rev 2009;30:557-85. 
7. Chen L, Magliano DJ, Zimmet PZ. The worldwide epidemiology of Type 2 diabetes mellitus present and future perspectives. Nat Rev Endocrinol 2011;8:228-36.

8. Olokoba AB, Obateru OA, Olokoba LB. Type 2 diabetes mellitus: A review of current trends. Oman Med J 2012;27:269-73.

9. Nair SS, Kavrekar V, Mishra A. In vitro studies on alpha amylase and alpha glucosidase inhibitory activities of selected plant extracts. Eur J Exp Biol 2013;3:128-32.

10. Peter SJ, Sabina EP. Global current trends in natural products for diabetes management: A review. Int J Pharm Pharm Sci 2016;8:20-8.

11. Yee HS, Fong NT. A review of the safety and efficacy of acarbose in diabetes mellitus. Pharmacotherapy 1996;16:792-805.

12. van de Laar FA. Alpha-glucosidase inhibitors in the early treatment of Type 2 diabetes. Vasc Health Risk Manag 2008;4:1189-95.

13. Moradi M, Mousavi S. Drug use evaluation of diabetes mellitus in nonhospitalized patients. Int J Pharm Pharm Sci 2016;8:337-41.

14. Zonoubi A, Perumal DV, Prasad P, Chandy V, Mafibaniasadi Z. Milk thistle-morphology, chemistry and pharmacological action. Int J Innov Pharm Sci Res 2019;7:14-40.

15. Kitchen DB, Decornez H, Furr JR, Bajorath J. Docking and scoring in virtual screening for drug discovery: Methods and applications. Nat Rev Drug Discov 2004;3:935-49.

16. Gilson MK, Zhou HX. Calculation of protein-ligand binding affinities. Annu Rev Biophys Biomol Struct 2007;36:21-42.

17. Qing X, Lee XY, De Raeymaeker J, Tame JR, Zhang KY, De Maeyer M, et al. Pharmacophore modeling: Advances, limitations, and current utility in drug discovery. J Recept Ligand Channel Res 2014;7:81-92.

18. Yang SY. Pharmacophore modeling and applications in drug discovery: Challenges and recent advances. Drug Discov Today 2010;15:444-50.

19. Mukesh B, Rakesh K. Molecular docking: A review. Int J Res Ayurveda Pharm 2011;2:1746-51.

20. Altschul SF, Gish W, Miller W, Myers EW, Lipman DJ. Basic local alignment search tool. J Mol Biol 1990;215:403-10.

21. Altschul SF, Madden TL, Schäffer AA, Zhang J, Zhang Z, Miller W, et al. Gapped BLAST and PSI-BLAST: A new generation of protein database search programs. Nucleic Acids Res 1997;25:3389-402.

22. Gibson TJ, Higgins DG. Multiple sequence alignment using ClustalW and ClustalX. Curr Protoc Bioinformatics 2002; Chapter 2:Unit 2.3.

23. Deshpande N, Addess KJ, Bluhm WF, Merino-Ott JC, Townsend-Merino $\mathrm{W}$, Zhang Q, et al. The RCSB protein data bank: A redesigned query system and relational database based on the mmCIF schema. Nucleic Acids Res 2005:33:D233-7.

24. Laskowski RA, MacArthur MW, Moss DS, Thornton JM. PROCHECK: A program to check the stereochemical quality of protein structures. J Appl Crystallogr 1993;26:283-91.

25. Gopalakrishnan K, Sowmiya G, Sheik SS, Sekar K. Ramachandran plot on the web (2.0). Protein Pept Lett 2007;14:669-71

26. Emsley P, Cowtan K. Coot: Model-building tools for molecular graphics. Acta Crystallogr D Biol Crystallogr 2004;60:2126-32.

27. Krissinel E, Henrick K. Secondary-structure matching (SSM), a new tool for fast protein structure alignment in three dimensions. Acta Crystallogr D Biol Crystallogr 2004;60:2256-68.

28. Tian W, Chen C, Lei X, Zhao J, Liang J. CASTp 3.0: Computed atlas of surface topography of proteins. Nucleic Acids Res 2018;46:W363-7.

29. Edelsbrunner H. Three-dimensional alpha shapes. ACM Trans Graph 1994;13:43-72.

30. Shape ID. The union of balls and its dual shape. Discrete Comput Geom 1995;13:415-40.

31. Edelsbrunner H, Shah NR. Incremental topological flipping works for regular triangulation. Algorithmica 1996;15:223-41.

32. Edelsbrunner H, Liang J, Fu P, Facello M. Measuring Proteins and Voids in Proteins. Vol. 1. Proceedings of the Twenty-Eighth Hawaii International Conference; 1995. p. 256.

33. Edelsbrunner H, Facello M, Liang J. On the definition and the construction of pockets in macromolecules. Discrete Appl Math 1998;88:83-102.

34. Lipinski CA, Lombardo F, Dominy BW, Feeney PJ. Experimental and computational approaches to estimate solubility and permeability in drug discovery and development settings. Adv Drug Deliv Rev 2001;46:3-26.

35. Cheng F, Li W, Zhou Y, Shen J, Wu Z, Liu G, et al. AdmetSAR: A comprehensive source and free tool for assessment of chemical ADMET properties. J Chem Inf Model 2012;52:3099-105.

36. Ertl P, Rohde B, Selzer P. Fast calculation of molecular polar surface area as a sum of fragment-based contributions and its application to the prediction of drug transport properties. J Med Chem 2000;43:3714-7.

37. Veber DF, Johnson SR, Cheng HY, Smith BR, Ward KW, Kopple KD, et al. Molecular properties that influence the oral bioavailability of drug candidates. J Med Chem 2002;45:2615-23.

38. Edwards BS, Bologa C, Young SM, Balakin KV, Prossnitz ER, Savchuck NP, et al. Integration of virtual screening with high-throughput flow cytometry to identify novel small molecule formylpeptide receptor antagonists. Mol Pharmacol 2005;68:1301-10.

39. Güner O, Clement O, Kurogi Y. Pharmacophore modeling and three dimensional database searching for drug design using catalyst: Recent advances pharmacophore modeling and three dimensional database searching for drug design using catalyst: Recent advances. Curr Med Chem 2004;11:763-71.

40. Navaneethakrishnan P, Prashantha CN, Boopathi S, Sabitha R, Mathan G. In silico design of Butea monosperma floral derived compounds and its inhibitory effect on $\beta$ catenin, gsk-3 $\beta$ and apc complex proteins in colorectal cancer. Int J Drug Discov 2013;5:191-7. 